\title{
Dyadic Green's Function Modifications for Obtaining Attenuation in Microstrip Transmission Layered Structures With Complex Media
}

\author{
Clifford M. Krowne, Senior Member, IEEE
}

\begin{abstract}
Rigorous derivation of the correction to the Green's function for a microstrip structure containing complex layered media is done for imperfect metallization. A hierarchy of formulas is found consistent with a full-wave electromagnetic code employing zero-thickness extent conductors for the guiding structure metal. At the top of the hierarchy are formulas that utilize new Green's functions of the structure, whereas at the bottom are formulas that are only dependent on the conductor geometry and material properties. Numerical examples are provided to show the sensitivity of the propagation constant attenuation to those elegantly simple formulas at the bottom of the hierarchy.
\end{abstract}

Index Terms-Attenuation constant, complex media, dyadic Green's function, microstrip, spectral-domain code.

\section{INTRODUCTION}

$\mathbf{S}_{\mathrm{n}}^{\mathrm{p}}$ PECTRAL-DOMAIN (SD) codes based upon the moment method, which solve the electromagnetic integral-equation problem for a multilayered structure, can place their prime focus on the material properties of the layers. Studies of the various anisotropies, nonreciprocities, crystallographic rotations, and biasing field orientations in ferromagnetic or ferroelectric materials can all be done with such codes [1], [2]. Theoretical investigations of this nature can be useful in deciding what structures to construct for integrated circuit applications [3] $]^{1}$. Device behavior will most often be decided by the resulting phase properties of the transmission structures (e.g., phase shifting, coupling, delay). However, being able to reasonably accurately predict the loss consequences of the imperfect metallizations used in actual devices may be necessary for the eventual development of working devices in circuits.

Full-wave electromagnetic codes employing zero-thickness extent conductors for the guiding structure metal can be modified to account for the finite conductivity of the metallization and thickness. The most straightforward modifications, which are self-consistent in that they avoid any perturbational approaches, will alter the structure interfacial dyadic Green's function [4] where the guiding metal is located, with an expression that only depends upon the metallization geometry

Manuscript received December 3, 2000. This work was supported by the Defense Advanced Projects Agency under the Frequency Agile Materials for Electronics Program.

The author is with Code 6850.3, Microwave Technology Branch, Electronics Science and Technology Division, Naval Research Laboratory, Washington, DC 20375-5347 USA (e-mail: krowne@estd.nrl.navy.mil).

Publisher Item Identifier S 0018-9480(02)00759-7.

${ }^{1}$ In [3], voltage bias labels were inadvertently reversed in Fig. 3. and conductivity. These modifications can be employed most broadly for different SD codes. These modifications can be shown to come from a more general class of modifications that require additional structure dyadic Green's functions at the interface. Governing equations that employ these additional structure dyadic Green's functions at the interface have a form that is applicable to different SD codes, but each code will require its own specific interfacial dyadic Green's functions to actually effect the modification. Thus, the more general approach cannot be as easily applied to different SD codes.

For situations where the metallizations are very thick compared to other structure geometric dimensions, where the metallization thicknesses are expected to significantly alter the basic device behavior, or when it is desired to obtain the nearly exact field distribution in the metallization vicinity, the approach discussed in this paper should not be used. Then other methods taking into account explicitly the metallization geometry and material characteristics should be used. It should be noted here that metallizations can be quite complicated, employing several layers of different conductivity materials, different surface roughness properties, varying edge shapes, and even different cross-sectional configurations. Appropriate two-dimensional methods include finite difference, finite element, mode matching, SD, method-of-lines, or some other approach, which either model the metal with surface impedance or interior metallization discretization (e.g. [5]-[21]²).

\section{GENERAL THEORY}

At the location where the guiding metal exists (if we are talking about a single interface)

$$
\mathbf{E}=\mathbf{E}_{d}+\mathbf{E}_{c} \text {. }
$$

$\mathbf{E}, \mathbf{E}_{d}$, and $\mathbf{E}_{c}$ are, respectively, the total electric-field vector at the interface, electric-field vector at the interface where there is a dielectric mismatch, and electric-field vector at the interface where there is a conductor. $\mathbf{E}_{d}$ and $\mathbf{E}_{c}$ are zero outside their existence range. Since we will test this relationship with surface currents, it is the following tangential form that is of greatest interest:

$$
\mathbf{E}_{\tan }=\mathbf{E}_{d, \tan }+\mathbf{E}_{c, \tan }
$$

${ }^{2}$ In [21], the first hyperbolic factor of $\mathbf{J}(x, 0)$ in (1) should read $\sinh \left(t^{\prime}-\right.$ $\left.z^{\prime}\right) / \sinh \left(t^{\prime}\right)$. Communicated by Rolf H. Jansen. 
Multiply the total electric field by a test surface current vector $\mathbf{J}_{s j}$ or its conjugate and integrate over the interfacial coordinate $x$. The result is

$$
\begin{aligned}
& \int_{0}^{b} \mathbf{E}_{\tan } \cdot \mathbf{J}_{s j} d x=\int_{0}^{b} \mathbf{E}_{d, \tan } \cdot \mathbf{J}_{s j} d x+\int_{0}^{b} \mathbf{E}_{c, \tan } \cdot \mathbf{J}_{s j} d x \\
& \int_{0}^{b} \mathbf{E}_{\tan } \cdot \mathbf{J}_{s j}^{*} d x=\int_{0}^{b} \mathbf{E}_{d, \tan } \cdot \mathbf{J}_{s j}^{*} d x+\int_{0}^{b} \mathbf{E}_{c, \tan } \cdot \mathbf{J}_{s j}^{*} d x
\end{aligned}
$$

where $b$ is the box width. Since $\mathbf{E}_{d}$ and $\mathbf{J}_{s j}$ or $\mathbf{J}_{s j}^{*}$ are complementary functions on the finite $x$-space, the first integral on the right-hand side is zero. Thus, we find

$$
\int_{0}^{b} \mathbf{E}_{\tan } \cdot \mathbf{J}_{s j} d x=\int_{0}^{b} \mathbf{E}_{c, \tan } \cdot \mathbf{J}_{s j} d x
$$

or

$$
\int_{0}^{b} \mathbf{E}_{\tan } \cdot \mathbf{J}_{s j} d x-\int_{0}^{b} \mathbf{E}_{c, \tan } \cdot \mathbf{J}_{s j} d x=0 .
$$

Each integral on the left-hand side may be subject to having Parseval's theorem applied, transforming each into either an integral or an infinite summation, depending on whether or not the transformed variable $k_{x}$ is continuous or discrete. Since the domain for $x$ is finite, $k_{x}$ must be discrete and it may be set dependent on the integer index $n$, i.e., $k_{x}=k_{x}(n)$. The relation becomes

$$
\int_{0}^{b} \mathbf{E}_{\tan } \cdot \mathbf{J}_{s j} d x-\frac{(-1)^{p}}{b} \sum_{n=-\infty}^{\infty} \tilde{\mathbf{E}}_{c, \tan }(n) \cdot \tilde{\mathbf{J}}_{s j}(n)=0
$$

Here, $p$ is the parity integer for the test basis function $\mathbf{J}_{s j}(p=0$ for even symmetry with respect to the $x$-axis, $p=1$ for odd symmetry). We have only transformed the second integral because there is a problem that must be addressed in how it is to be evaluated. $\tilde{\mathbf{E}}_{c, \tan }(n)$ really must represent some transform averaging over the thickness of the conductor, say, over slices taken at different $y$ values $y \in[0, t]$. This averaging may be denoted by $\left\langle\tilde{\mathbf{E}}_{c, \tan }(n, y)\right\rangle_{\text {av }}$ with the assignment $\tilde{\mathbf{E}}_{c, \tan }(n) \rightarrow$ $\tilde{\mathbf{E}}_{c, \tan ; \mathbf{a v}}(n)=\left\langle\tilde{\mathbf{E}}_{c, \tan }(n, y)\right\rangle$. This averaging is not unique. The transform of the field in the conductor is

$$
\begin{aligned}
\tilde{\mathbf{E}}_{c, \tan }(n, y) & =\int_{0}^{b} \mathbf{E}_{c, \tan }(x, y) e^{-j k_{x} x} d x \\
& =\int_{-w / 2}^{w / 2} \mathbf{E}_{c, \tan }(x, y) e^{-j k_{x} x} d x
\end{aligned}
$$

where the second line has shifted the $x$-coordinate to the strip center and limits the integration to where the conductor field exists. Applying the averaging operator to this expression

$$
\begin{aligned}
\tilde{\mathbf{E}}_{c, \tan ; \mathbf{a v}}(n) & =\left\langle\tilde{\mathbf{E}}_{c, \tan }(n, y)\right\rangle_{\mathrm{av}} \\
& =\int_{-w / 2}^{w / 2}\left\langle\mathbf{E}_{c, \tan }(x, y)\right\rangle_{\mathbf{a v}} e^{-j k_{x} x} d x .
\end{aligned}
$$

Inserting the average into (5)

$$
\begin{array}{r}
\int_{0}^{b} \mathbf{E}_{\tan } \cdot \mathbf{J}_{s j} d x-\frac{(-1)^{p}}{b} \sum_{n=-\infty}^{\infty}\left\langle\tilde{\mathbf{E}}_{c, \tan }(n, y)\right\rangle_{\text {av }} \\
\cdot \tilde{\mathbf{J}}_{s j}(n)=0 .
\end{array}
$$

Now applying Parseval's theorem to the first integral in (8)

$$
\sum_{n=-\infty}^{\infty} \tilde{\mathbf{E}}_{\tan } \cdot \tilde{\mathbf{J}}_{s j}(n)-\sum_{n=-\infty}^{\infty}\left\langle\tilde{\mathbf{E}}_{c, \tan }(n, y)\right\rangle_{\mathrm{av}} \cdot \tilde{\mathbf{J}}_{s j}(n)=0
$$

or

$$
\begin{aligned}
& \sum_{n=-\infty}^{\infty}\left[\tilde{\mathbf{E}}_{\tan }-\left\langle\tilde{\mathbf{E}}_{c, \tan }(n, y)\right\rangle_{\mathrm{av}}\right] \cdot \tilde{\mathbf{J}}_{s j}(n)=0, \\
& \quad j=1,2, \ldots, n_{x}+n_{z} .
\end{aligned}
$$

Indexing for $\mathbf{J}_{s j}$ is based upon a single index notation, which allows discrimination between different current components. This is done by setting

$$
\begin{array}{ll}
\tilde{\mathbf{J}}_{s i}(n)=\tilde{J}_{s i x}(n) \hat{x}=\left[\begin{array}{c}
\tilde{J}_{s i x}(n) \\
0
\end{array}\right], \quad i=1,2, \ldots, n_{x} \\
\tilde{\mathbf{J}}_{s k}(n)=\tilde{J}_{s k i z}(n) \hat{z}=\left[\begin{array}{c}
0 \\
\tilde{J}_{s k i z}(n)
\end{array}\right], \quad k=1,2, \ldots, n_{z}
\end{array}
$$

These are combined into a single indexing vector scheme

$$
\tilde{\mathbf{J}}_{s j}(n)=\left[\begin{array}{c}
\tilde{J}_{s j x}(n) \\
\tilde{J}_{s j z}(n)
\end{array}\right], \quad j=1,2, \ldots, n_{x}+n_{z}
$$

where

$$
\begin{array}{r}
\tilde{J}_{s j x}(n) \\
= \begin{cases}\tilde{J}_{s i x}(n), & j=i=1,2, \ldots, n_{x} \\
0, \quad j=n_{x}+1, n_{x}+2, \ldots, n_{x}+n_{z}\end{cases} \\
\tilde{J}_{s j z}(n) \\
= \begin{cases}0, & j=1,2, \ldots, n_{x} \\
\tilde{J}_{s k z}(n), & j=k+i_{\max }=n_{x}+1, n_{x} \\
+2, \ldots, n_{x}+n_{z} .\end{cases}
\end{array}
$$

Surface current is then expanded as follows:

$$
\tilde{\mathbf{J}}_{s}(n)=\sum_{j=1}^{n_{x}+n_{z}} a_{j} \tilde{\mathbf{J}}_{s j}(n)
$$

\section{REPRESENTATION OF CONDUCTOR BY SURFACE IMPEDANCE}

In order to develop (10) further, evaluation of $\left\langle\tilde{\mathbf{E}}_{c, \tan }(n, y)\right\rangle_{\text {av }}$ must be addressed. Here, we will consider the conductor to be thick enough that it can be viewed as being composed of an upper piece of one material having a surface impedance $Z_{s u}$ and a lower piece having $Z_{s l}$. The electric field at either surface is given by

$$
\tilde{\mathbf{E}}_{s}=Z_{s}\left(\hat{n} \times \tilde{\mathbf{H}}_{s}\right)
$$




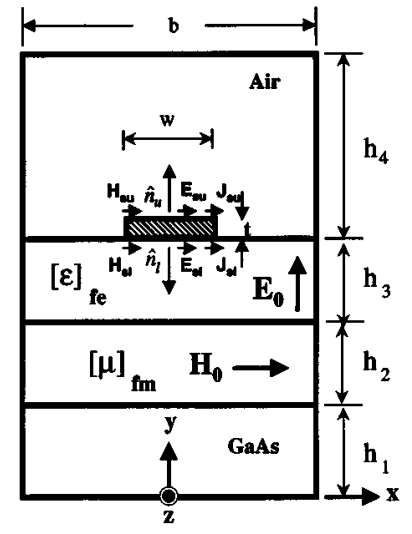

Fig. 1. Cross-sectional diagram of an example complex layered structure propagating waves in the $z$-direction. Here, a strip conductor is over a multilayered substrate composed of anisotropic ferroelectric and ferromagnetic materials biased with static vector electric and magnetic fields. Conductor cross section above and below is labeled with relevant tangential fields analyzed in the text.

Here, $\hat{n}$ is the unit surface normal pointing from the metal into the upper or lower region. Thus (see Fig. 1 for location of surface tangential fields and normals of the strip)

$$
\begin{aligned}
\left\langle\tilde{\mathbf{E}}_{c, \tan }(n, y)\right\rangle_{\mathrm{av}} & =\left\langle\tilde{\mathbf{E}}_{c, \tan }(n, y=t), \tilde{\mathbf{E}}_{c, \tan }(n, y=0)\right\rangle_{\mathrm{av}} \\
& =\left\langle\tilde{\mathbf{E}}_{u, \tan }, \tilde{\mathbf{E}}_{l, \tan }\right\rangle_{\mathrm{av}} .
\end{aligned}
$$

In (16), subscripts tan have been added to emphasize the tangential nature of the resultant electric-field components in (15). It is apparent that the cross product does not so require the $\mathbf{H}_{s}$-fields to be identified, but such identification is useful in the soon-to-follow relation of the surface magnetic fields $\mathbf{H}_{s}$ to the surface currents. Surface electric fields may be written as

$$
\tilde{\mathbf{E}}_{s}=\sum_{i=1}^{n} a_{i} \tilde{\mathbf{E}}_{s i}
$$

This field is the result of the superposition of all the terms in the expansion (14), operating through the propagation structure Green's function. Inserting (15) and (17) into (16)

$$
\begin{aligned}
& \left\langle\tilde{\mathbf{E}}_{c, \tan }(n, y)\right\rangle_{\mathrm{av}} \\
& =\left\langle\sum_{i=1}^{n} a_{i} \tilde{\mathbf{E}}_{s u i}, \sum_{i=1}^{n} a_{i} \tilde{\mathbf{E}}_{s l i}\right\rangle_{\mathrm{av}} \\
& =\sum_{i=1}^{n}\left\langle\tilde{\mathbf{E}}_{s u i}, \tilde{\mathbf{E}}_{s l i}\right\rangle_{\mathrm{av}} a_{i} \\
& =\sum_{i=1}^{n}\left\langle Z_{s u}\left(\hat{n}_{u} \times \tilde{\mathbf{H}}_{s u i}\right), Z_{s l}\left(\hat{n}_{l} \times \tilde{\mathbf{H}}_{s l i}\right)\right\rangle_{\mathrm{av}} a_{i} \\
& =\sum_{i=1}^{n} \hat{n}_{u} \times\left\langle Z_{s u} \tilde{\mathbf{H}}_{s u i},-Z_{s l} \tilde{\mathbf{H}}_{s l i}\right\rangle_{\mathrm{av}} a_{i} .
\end{aligned}
$$

Here, the linearity property of the averaging operator \langle\rangle$_{\mathbf{a v}}$ has been used to extract the coefficients $a_{i}$ and the vector $\hat{n}_{u}=\hat{y}$. Upper and lower surface magnetic fields may be related by

$$
\hat{n}_{u} \times \tilde{\mathbf{H}}_{s u}=\hat{n}_{u} \times \tilde{\mathbf{H}}_{s l}+\tilde{\mathbf{J}}_{s} .
$$

Relation (19) is exact at a single interface, thus, it must be viewed as an approximation, somewhat convincingly since the fields in it are tied together by the single surface current characteristic of infinitely thin-metal Green's function SD solvers. Equation (19) may be employed for basis function $i$, and used to eliminate unknown upper field $\tilde{\mathbf{H}}_{\text {sui }}$ by using a magnetic dyadic Green's function for the unknown lower field $\tilde{\mathbf{H}}_{s l i}$, i.e.,

$$
\tilde{\mathbf{H}}_{s l i}=\mathbf{G}_{\mathbf{H}_{s} \mathbf{J}} \tilde{\mathbf{J}}_{s i}
$$

which is already available for the structure.

Putting (19) and (20) into (18)

$$
\begin{aligned}
\left\langle\tilde{\mathbf{E}}_{c, \tan }(n, y)\right\rangle_{\mathrm{av}} & \\
= & \sum_{i=1}^{n}\left\langle Z_{s u}\left(\hat{n}_{u} \times \tilde{\mathbf{H}}_{s l i}+\tilde{\mathbf{J}}_{s i}\right), Z_{s l}\left(\hat{n}_{l} \times \tilde{\mathbf{H}}_{s l i}\right)\right\rangle_{\mathrm{av}} a_{i} \\
= & \sum_{i=1}^{n}\left\langle Z_{s u}\left(\hat{n}_{u} \times \mathbf{G}_{\mathbf{H}_{s} \mathbf{J}} \tilde{\mathbf{J}}_{s i}+\tilde{\mathbf{J}}_{s i}\right),\right. \\
& \left.Z_{s l}\left(\hat{n}_{l} \times \mathbf{G}_{\mathbf{H}_{s} \mathbf{J}} \tilde{\mathbf{J}}_{s i}\right)\right\rangle_{\mathrm{av}} a_{i} .
\end{aligned}
$$

The effective surface electric field $\tilde{\mathbf{E}}_{s i}^{\prime}$ in the testing expression (10) can be written as

$$
\begin{aligned}
& \tilde{\mathbf{E}}_{s i}^{\prime}= \tilde{\mathbf{E}}_{s i}-\left\langle\mathbf{E}_{c, \tan }(n, y)\right\rangle_{\mathrm{av}, i} \\
&= \mathbf{G}_{\mathbf{E}_{s} \mathbf{J}_{s i}} \tilde{\mathbf{J}}_{s i}-\left\langle Z_{s u}\left(\hat{n}_{u} \times \mathbf{G}_{\mathbf{H}_{s} \mathbf{J}_{s i}} \tilde{\mathbf{J}}_{s i}+\tilde{\mathbf{J}}_{s i}\right),\right. \\
&\left.-Z_{s l}\left(\hat{n}_{u} \times \mathbf{G}_{\mathbf{H}_{s} \mathbf{J}_{s i}} \tilde{\mathbf{J}}_{s i}\right)\right\rangle_{\mathbf{a v}} \\
&= \mathbf{G}_{\mathbf{E}_{s} \mathbf{J}_{s i}} \tilde{\mathbf{J}}_{s i}-\left\langle Z_{s u}\left(\hat{n}_{u} \times \mathbf{G}_{\mathbf{H}_{s} \mathbf{J}_{s i}}+1\right),\right. \\
&-Z_{s l}\left(\hat{n}_{u} \times \mathbf{G}_{\left.\mathbf{H}_{s} \mathbf{J}_{s i}\right)}\right) \tilde{\mathbf{J}}_{s i} \\
&=\left[\mathbf{G}_{\mathbf{E}_{s} \mathbf{J}_{s i}}-\left\langle Z_{s u}\left(\hat{n}_{u} \times \mathbf{G}_{\mathbf{H}_{s} \mathbf{J}_{s i}}+1\right),\right.\right. \\
& \quad-Z_{s l}\left(\hat{n}_{u} \times \mathbf{G}_{\left.\left.\mathbf{H}_{s} \mathbf{J}_{s i}\right)\right\rangle_{\mathbf{a v}}}\right] \tilde{\mathbf{J}}_{s i}
\end{aligned}
$$

where the testing expression (10) becomes

$$
\begin{gathered}
\sum_{n=-\infty}^{\infty} \sum_{i=1}^{n_{x}+n_{z}} a_{i} \tilde{\mathbf{E}}_{s i}^{\prime}(n) \cdot \tilde{\mathbf{J}}_{s j}(n)=0, \\
j=1,2, \ldots, n_{x}+n_{z}
\end{gathered}
$$

The above reduction is valid if the averaging operation is strictly linear, in actuality required in a rigorous sense since the Green's function approach is a linear process. Extraction of surface current has also depended upon the property that the ordering of the products inside the averaging operator does not matter. That is, the unit normal vector crossed into the dyadic magnetic Green's function, then multiplied by the surface current, yields the same result as the dyadic magnetic Green's function multiplied by the surface current, followed by a cross-product multiplication by the unit normal vector. Equation (22) is a rather remarkable result, as it allows us to write down by inspection the new Green's 
function for the finite-sized and finite-conductivity metal strip in our anisotropic layered structure

$$
\begin{array}{r}
\mathbf{G}_{\mathbf{E}_{s} \mathbf{J}_{s i}=}^{\prime} \mathbf{G}_{\mathbf{E}_{s} \mathbf{J}_{s i}}-\left\langle Z_{s u}\left(\hat{n}_{u} \times \mathbf{G}_{\mathbf{E}_{s} \mathbf{J}_{s i}}+1\right),\right. \\
-Z_{s l}\left(\hat{n}_{u} \times \mathbf{G}_{\left.\left.\mathbf{E}_{s} \mathbf{J}_{s i}\right)\right\rangle_{\mathbf{a v}}} .\right.
\end{array}
$$

We will not pursue this approach further, other than to say that, although the form has been presented, it does not prove that, in fact, such a closed-form representation can be found. Instead, in order to have some specific rules for constructing the average, resort to a procedure found in [22]. For any two spectral vectors $\tilde{\mathbf{A}}$ and $\tilde{\mathbf{B}}$, the average is

$$
\langle\tilde{\mathbf{A}}, \tilde{\mathbf{B}}\rangle_{\mathrm{av}}=\frac{A_{x}\left|A_{x}\right|+B_{x}\left|B_{x}\right|}{\left|A_{x}\right|+\left|B_{x}\right|} \hat{x}+\frac{A_{z}\left|A_{z}\right|+B_{z}\left|B_{z}\right|}{\left|A_{z}\right|+\left|B_{z}\right|} \hat{z} .
$$

Inversion of the magnitudes in (25) will make the averaging operator nonlinear, showing that such a construction to account for finite thickness and conductivity of the metal must be an approximation since the actual problem is linear in the driving current $\tilde{\mathbf{J}}_{s}$. Nevertheless, we will enlist this formula to have something definite to discuss.

Therefore, write the conductor field as

$$
\begin{aligned}
& \left\langle\tilde{\mathbf{E}}_{c, \tan }(n, y)\right\rangle_{\mathrm{av}, i} \\
& =\left\langle\tilde{\mathbf{E}}_{u i, \tan }, \tilde{\mathbf{E}}_{l i, \tan }\right\rangle_{\mathrm{av}} \\
& =\left\langle Z_{s u}\left(\hat{n}_{u} \times \tilde{\mathbf{H}}_{s u i}\right),-Z_{s l}\left(\hat{n}_{u} \times \tilde{\mathbf{H}}_{s l i}\right)\right\rangle_{\mathrm{av}} \\
& =\left\langle Z_{s u}\left(\hat{y} \times\left[\begin{array}{c}
-\tilde{J}_{s u z i} \\
\tilde{J}_{s u x i}
\end{array}\right]\right),-Z_{s l}\left(\hat{y} \times\left[\begin{array}{c}
\tilde{J}_{s l z i} \\
-\tilde{J}_{s l x i}
\end{array}\right]\right)\right\rangle_{\mathrm{av}} \\
& =\left\langle Z_{s u} \tilde{\mathbf{J}}_{s u i}, Z_{s l} \tilde{\mathbf{J}}_{s l i}\right\rangle_{\text {av }}
\end{aligned}
$$

which comes about by using the relationship between the magnetic fields at the lower and upper surfaces and their respective surface currents. Using the averaging recipe (25)

$$
\begin{aligned}
& \left\langle\tilde{\mathbf{E}}_{c, \tan }(n, y)\right\rangle_{\mathrm{av}, i} \\
& =\frac{Z_{s u} \tilde{J}_{s u x i}\left|Z_{s u} \tilde{J}_{s u x i}\right|+Z_{s l} \tilde{J}_{s l x i}\left|Z_{s l} \tilde{J}_{s l x i}\right|}{\left|Z_{s u} \tilde{J}_{s u x i}\right|+\left|Z_{s l} \tilde{J}_{s l x i}\right|} \hat{x} \\
& \\
& +\frac{Z_{s u} \tilde{J}_{s u z i}\left|Z_{s u} \tilde{J}_{s u z i}\right|+Z_{s l} \tilde{J}_{s l z i}\left|Z_{s l} \tilde{J}_{s l z i}\right|}{\left|Z_{s u} \tilde{J}_{s u z i}\right|+\left|Z_{s l} \tilde{J}_{s l z i}\right|} \hat{z} .
\end{aligned}
$$

Surface components in (27) are related to the interfacial expanded surface current by

$$
\tilde{J}_{s x i}=\tilde{J}_{s u x i}+\tilde{J}_{s l x i} \quad \tilde{J}_{s z i}=\tilde{J}_{s u z i}+\tilde{J}_{s l z i} .
$$

Note that the right-hand-side current components in (28) can be related to the magnetic fields by

$$
\tilde{\mathbf{H}}_{s u i}=\left[\begin{array}{c}
-\tilde{J}_{s u z i} \\
\tilde{J}_{s u x i}
\end{array}\right] \quad \tilde{\mathbf{H}}_{s l i}=\left[\begin{array}{c}
\tilde{J}_{s l z i} \\
-\tilde{J}_{s l x i}
\end{array}\right]
$$

and these magnetic fields to the surface current through (19) and (20). This provides a complicated method for evaluating (27) in terms of $\tilde{\mathbf{J}}_{s i}$, and we see immediately that indeed the $\left\langle\tilde{\mathbf{E}}_{c, \tan }(n, y)\right\rangle_{\mathrm{av}, i}$ quantity is nonlinearly related to $\tilde{\mathbf{J}}_{s i}$. With (27), we can follow the procedure used in getting $\tilde{\mathbf{E}}_{s i}^{\prime}$ in (22) and produce what can only be stated as an improper Green's function, as shown in (30), at the bottom of this page. This type of Green's function only has meaning when used to reconstruct the $\tilde{\mathbf{E}}_{s i}^{\prime}$ field [which is used in the testing procedure (23)], and it is not unique-only when multiplied by $\widetilde{\mathbf{J}}_{s i}$ is the result unique. $\mathbf{G}_{\mathbf{E}_{s} \mathbf{J}_{s i}}^{\prime}$ has been shown to demonstrate the intrinsic complexity of trying to get a tractable modified Green's function using the averaging procedure in (25).

Consider what happens to (30) when the upper and lower surface impedances are equal, i.e., $Z_{s u}=Z_{s l}$, as shown in (31), at the bottom of the following page. When $Z_{s u}$ and $Z_{s l}$ are taken to be real, we get (32), shown at the bottom of the following page. Next, look at how (31) for $Z_{s u}=Z_{s l}$ simplifies when

$$
\tilde{\mathbf{J}}_{s u i}+\tilde{\mathbf{J}}_{s l i}=\tilde{\mathbf{J}}_{s i} \quad \tilde{\mathbf{J}}_{s u i}-\tilde{\mathbf{J}}_{s l i}=f_{J} \tilde{\mathbf{J}}_{s i}
$$

where it is assumed that $f_{J}$ is an $i$ index independent. We expect $f_{J}<0$, and substituting

$$
\tilde{\mathbf{J}}_{s u i}=\frac{1+f_{J}}{2} \tilde{\mathbf{J}}_{s i} \quad \tilde{\mathbf{J}}_{s l i}=\frac{1-f_{J}}{2} \tilde{\mathbf{J}}_{s i}
$$

into (31) yields

$$
\begin{aligned}
& \mathbf{G}_{\mathbf{E}_{s} \mathbf{J}_{s i}}^{\prime} \\
& \quad=\mathbf{G}_{\mathbf{E}_{s} \mathbf{J}_{s i}}-\left[\begin{array}{cc}
\frac{Z_{s}}{2}\left(1+f_{J}^{2}\right) & 0 \\
0 & \frac{Z_{s}}{2}\left(1+f_{J}^{2}\right)
\end{array}\right] \\
& =\left[\begin{array}{cc}
G_{\mathbf{E}_{s} \mathbf{J}_{s i} x x}-\frac{Z_{s}}{2}\left(1+f_{J}^{2}\right) & G_{\mathbf{E}_{s} \mathbf{J}_{s i} x z} \\
G_{\mathbf{E}_{s} \mathbf{J}_{s i} z x} & G_{\mathbf{E}_{s} \mathbf{J}_{s i} z z}-\frac{Z_{s}}{2}\left(1+f_{J}^{2}\right)
\end{array}\right] .
\end{aligned}
$$

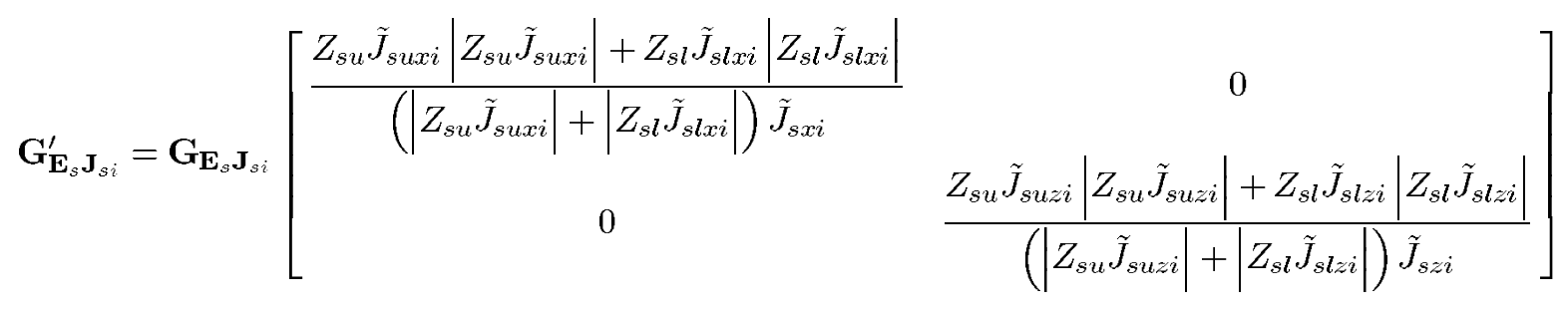


Diagonal correction element $Z_{s}\left(1+f_{J}^{2}\right) / 2$ reduces to $Z_{s}$ when there is no top surface current $\left(f_{J}=-1\right)$ or bottom surface current $\left(f_{J}=1\right)$ and to $Z_{s} / 2$ when the top and bottom surface currents are equal $\left(f_{J}=0\right)$.

\section{TANGENTIAL-FIELD BEHAVIOR IN STRIP-EXPLICIT FIELD DETERMINATION}

Detailed field behavior within the strip metal can be found by utilizing the Maxwell curl equations

$$
\begin{aligned}
\nabla \times \mathbf{E} & =-\mu_{m} \frac{\partial \mathbf{H}}{\partial t} \\
\nabla \times \mathbf{H} & =-\varepsilon_{m} \frac{\partial \mathbf{E}}{\partial t}+\sigma_{m} \mathbf{E} .
\end{aligned}
$$

For harmonic conditions where the time variation is $e^{j \omega t}$, these equations take the form

$$
\begin{aligned}
& \left(\frac{\partial E_{z}}{\partial y}-\frac{\partial E_{y}}{\partial z}\right) \hat{x}+\left(\frac{\partial E_{x}}{\partial z}-\frac{\partial E_{z}}{\partial x}\right) \hat{y}+\left(\frac{\partial E_{y}}{\partial x}-\frac{\partial E_{x}}{\partial y}\right) \hat{z} \\
& =-j \omega \mu_{m} \mathbf{H} \\
& \left(\frac{\partial H_{z}}{\partial y}-\frac{\partial H_{y}}{\partial z}\right) \hat{x}+\left(\frac{\partial H_{x}}{\partial z}-\frac{\partial H_{z}}{\partial x}\right) \hat{y}+\left(\frac{\partial H_{y}}{\partial x}-\frac{\partial H_{x}}{\partial y}\right) \hat{z} \\
& =\left(j \omega \varepsilon_{m}+\sigma_{m}\right) \mathbf{E} .
\end{aligned}
$$

If $L, w \gg \delta$ for all metals encountered, the strip will look like a large uniform sheet in the $x z$-plane. Thus, $\partial / \partial x \rightarrow 0$ and $\partial / \partial z \rightarrow 0$ and

$$
\begin{aligned}
& \frac{\partial E_{z}}{\partial y} \hat{x}-\frac{\partial E_{x}}{\partial y} \hat{z}=-j \omega \mu_{m} \mathbf{H} \\
& \frac{\partial H_{z}}{\partial y} \hat{x}-\frac{\partial H_{x}}{\partial y} \hat{z}=\left(j \omega \varepsilon_{m}+\sigma_{m}\right) \mathbf{E} .
\end{aligned}
$$

In order to learn what the tangential components do when traveling through the strip in the normal $y$-direction, we will exclude normal $E_{y}$ - or $H_{y}$-field components, making the waves $\mathrm{TEM}_{y}$. Equation (38) becomes for the $\left(E_{z}, H_{x}\right)$ pair

$$
\begin{aligned}
\frac{\partial E_{z}}{\partial y}+j \omega \mu_{m} H_{x} & =0 \\
\frac{\partial H_{x}}{\partial y}+\left(j \omega \varepsilon_{m}+\sigma_{m}\right) E_{z} & =0 .
\end{aligned}
$$

For the $\left(E_{x}, H_{z}\right)$ pair, (38) become

$$
\begin{aligned}
\frac{\partial E_{x}}{\partial y}-j \omega \mu_{m} H_{z} & =0 \\
\frac{\partial H_{z}}{\partial y}-\left(j \omega \varepsilon_{m}+\sigma_{m}\right) E_{x} & =0 .
\end{aligned}
$$

Adding these two equations together in a vector fashion

$$
\begin{array}{r}
\frac{\partial\left(E_{z} \hat{z}+E_{x} \hat{x}\right)}{\partial y}+j \omega \mu_{m}\left(H_{x} \hat{z}-H_{z} \hat{x}\right)=0 \\
\frac{\partial\left(H_{x} \hat{x}+H_{z} \hat{z}\right)}{\partial y}+\left(j \omega \varepsilon_{m}+\sigma_{m}\right)\left[\left(E_{z} \hat{x}-E_{x} \hat{z}\right)\right]=0 .
\end{array}
$$

These may be rewritten as

$$
\frac{\partial \mathbf{E}_{\tan }}{\partial y}+j \omega \mu_{m}\left(-H_{x} \hat{y} \times \hat{x}-H_{z} \hat{y} \times \hat{z}\right)=0
$$

$$
\frac{\partial \mathbf{H}_{\mathrm{tan}}}{\partial y}+\left(j \omega \varepsilon_{m}+\sigma_{m}\right)\left[\left(E_{z} \hat{y} \times \hat{z}+E_{x} \hat{y} \times \hat{x}\right)\right]=0 .
$$

Factoring out $\hat{y}$ enables complete representation of these partial differential equations in terms of field vectors

$$
\begin{array}{r}
\frac{\partial \mathbf{E}_{\tan }}{\partial y}-j \omega \mu_{m} \hat{y} \times \mathbf{H}_{\tan }=0 \\
\frac{\partial \mathbf{H}_{\tan }}{\partial y}+\left(j \omega \varepsilon_{m}+\sigma_{m}\right) \hat{y} \times \mathbf{E}_{\tan }=0 .
\end{array}
$$

$$
\mathbf{G}_{\mathbf{E}_{s} \mathbf{J}_{s i}}^{\prime}=\mathbf{G}_{\mathbf{E}_{s} \mathbf{J}_{s i}}-Z_{s}\left[\begin{array}{cc}
\frac{\tilde{J}_{s u x i}\left|\tilde{J}_{s u x i}\right|+\tilde{J}_{s l x i}\left|\tilde{J}_{s l x i}\right|}{\left(\left|\tilde{J}_{s u x i}\right|+\left|\tilde{J}_{s l x i}\right|\right) \tilde{J}_{s x i}} & 0 \\
0 & \frac{\tilde{J}_{s u z i}\left|\tilde{J}_{s u z i}\right|+\tilde{J}_{s l z i}\left|\tilde{J}_{s l z i}\right|}{\left(\left|\tilde{J}_{s u z i}\right|+\left|\tilde{J}_{s l z i}\right|\right) \tilde{J}_{s z i}}
\end{array}\right]
$$

$$
\left.\frac{Z_{s u}^{2} \tilde{J}_{s u z i}\left|\tilde{J}_{s u z i}\right|+Z_{s l}^{2} \tilde{J}_{s l z i}\left|\tilde{J}_{s l z i}\right|}{\left(Z_{s u}\left|\tilde{J}_{s u z i}\right|+Z_{s l}\left|\tilde{J}_{s l z i}\right|\right) \tilde{J}_{s z i}}\right]
$$


Taking the partial derivative of (43a) and inserting (43b) into it gives

$$
\frac{\partial^{2} \mathbf{E}_{\mathrm{tan}}}{\partial y^{2}}+j \omega \mu_{m}\left(j \omega \varepsilon_{m}+\sigma_{m}\right) \hat{y} \times \hat{y} \times \mathbf{E}_{\mathrm{tan}}=0
$$

or

$$
\frac{\partial^{2} \mathbf{E}_{\mathrm{tan}}}{\partial y^{2}}-j \omega \mu_{m}\left(j \omega \varepsilon_{m}+\sigma_{m}\right) \mathbf{E}_{\mathrm{tan}}=0
$$

because $\hat{y} \times \hat{y} \times \mathbf{E}=(\hat{y} \cdot \mathbf{E}) \hat{y}-(\hat{y} \cdot \hat{y}) \mathbf{E}=E_{y} \hat{y}-\mathbf{E}=$ $-\mathbf{E}_{\text {tan }}$ holds. A similar statement may be made for the $\mathbf{H}$ field as follows:

$$
\frac{\partial^{2} \mathbf{H}_{\mathrm{tan}}}{\partial y^{2}}-j \omega \mu_{m}\left(j \omega \varepsilon_{m}+\sigma_{m}\right) \mathbf{H}_{\mathrm{tan}}=0 .
$$

Solutions to (45) and (46) are

$$
\begin{aligned}
\mathbf{E}_{\tan }(y) & =\mathbf{A}_{e} \cosh \gamma y+\mathbf{B}_{e} \sinh \gamma y \\
\mathbf{H}_{\tan }(y) & =\mathbf{A}_{h} \cosh \gamma y+\mathbf{B}_{h} \sinh \gamma y \\
\gamma & =\sqrt{j \omega \mu_{m}\left(j \omega \varepsilon_{m}+\sigma_{m}\right)} .
\end{aligned}
$$

Boundary conditions at $y=0$ and $y=t$ are

$$
\begin{array}{lll}
\mathbf{E}_{\tan }(y)=\mathbf{E}_{s l} & \mathbf{H}_{\tan }(y)=\mathbf{H}_{s l}, & y=0 \\
\mathbf{E}_{\tan }(y)=\mathbf{E}_{s u} & \mathbf{H}_{\tan }(y)=\mathbf{H}_{s u}, & y=t
\end{array}
$$

Applying (48a) to (47) produces

$$
\begin{aligned}
& \mathbf{A}_{e}=\mathbf{E}_{s l} \\
& \mathbf{A}_{h}=\mathbf{H}_{s l} .
\end{aligned}
$$

Placing (47) into (43a) and (43b) gives, respectively, at $y=0$

$$
\begin{aligned}
\mathbf{B}_{e} & =\frac{j \omega \mu_{m}}{\gamma} \hat{y} \times \mathbf{A}_{h}=\eta_{m} \hat{y} \times \mathbf{H}_{s l} \\
\mathbf{B}_{h} & =-\frac{\sigma_{m}+j \omega \varepsilon_{m}}{\gamma} \hat{y} \times \mathbf{A}_{e}=-\frac{1}{\eta_{m}} \hat{y} \times \mathbf{E}_{s l} \\
\eta_{m} & =\frac{j \omega \mu_{m}}{\gamma}
\end{aligned}
$$

Substitution of (49) and (50) into (47) produces the field solution within the metal strip

$$
\begin{aligned}
\mathbf{E}_{\tan }(y) & =\mathbf{E}_{s l} \cosh \gamma y+\eta_{m} \hat{y} \times \mathbf{H}_{s l} \sinh \gamma y \\
\mathbf{H}_{\tan }(y) & =\mathbf{H}_{s l} \cosh \gamma y-\frac{1}{\eta_{m}} \hat{y} \times \mathbf{E}_{s l} \sinh \gamma y
\end{aligned}
$$

Now we want to relate the upper and lower fields. This is done by applying boundary conditions (48b) to (51) as follows:

$$
\begin{aligned}
\mathbf{E}_{s u} & =\mathbf{E}_{s l} \cosh \gamma t+\eta_{m} \hat{y} \times \mathbf{H}_{s l} \sinh \gamma t \\
\mathbf{H}_{s u} & =\mathbf{H}_{s l} \cosh \gamma t-\frac{1}{\eta_{m}} \hat{y} \times \mathbf{E}_{s l} \sinh \gamma t
\end{aligned}
$$

Next, let us convert these equations into an impedance format. Solve (52b) for the normal times electric-field cross-product

$$
\hat{y} \times \mathbf{E}_{s l}=-\frac{\eta_{m}}{\sinh \gamma t} \mathbf{H}_{s u}+\eta_{m} \operatorname{coth} \gamma t \mathbf{H}_{s l} .
$$

Multiply (53) by $-\hat{y}$ to get the lower electric field

$$
-\hat{y} \times \hat{y} \times \mathbf{E}_{s l}=-\hat{y} \times\left[-\frac{\eta_{m}}{\sinh \gamma t} \mathbf{H}_{s u}+\eta_{m} \operatorname{coth} \gamma t \mathbf{H}_{s l}\right]
$$

or

$$
\mathbf{E}_{s l}=\hat{n}_{l} \times\left[-\frac{\eta_{m}}{\sinh \gamma t} \mathbf{H}_{s u}+\eta_{m} \operatorname{coth} \gamma t \mathbf{H}_{s l}\right] .
$$

Place (55a) into (52a) to obtain the upper electric field

$$
\begin{aligned}
\mathbf{E}_{s u}=\hat{y} \times & \left\{\eta_{m} \mathbf{H}_{s u} \operatorname{coth} \gamma t+\eta_{m} \mathbf{H}_{s l}\right. \\
& \times[-\operatorname{coth} \gamma t \cosh \gamma t+\sinh \gamma t]\} \\
= & \hat{n}_{u} \times\left\{\eta_{m} \operatorname{coth} \gamma t \mathbf{H}_{s u}-\frac{\eta_{m}}{\sinh \gamma t} \mathbf{H}_{s l}\right\} .
\end{aligned}
$$

Equation (55a) and (55b) may be recast in an explicit impedance form

$$
\begin{aligned}
\mathbf{E}_{s u} & =\hat{n}_{u} \times\left[Z_{u u} \mathbf{H}_{s u}+Z_{u l} \mathbf{H}_{s l}\right] \\
\mathbf{E}_{s l} & =\hat{n}_{l} \times\left[Z_{l u} \mathbf{H}_{s u}+Z_{l l} \mathbf{H}_{s l}\right] \\
Z_{u u} & =Z_{l l}=\eta_{m} \operatorname{coth} \gamma t \\
Z_{u l} & =Z_{l u}=-\frac{\eta_{m}}{\sinh \gamma t} .
\end{aligned}
$$

For metals like copper, silver, and gold, it is indeed true that $\sigma_{m} \gg j \omega \varepsilon_{m}$ [23], making (47c) and (50c) become

$$
\begin{gathered}
\gamma \rightarrow \frac{1+j}{\delta} \\
\delta=\frac{1}{\sqrt{\pi f \mu_{m} \sigma_{m}}}=\frac{2.09}{\sqrt{f(\mathrm{GHz})}} \sqrt{\frac{\sigma_{\mathrm{Cu}}}{\sigma_{m}}} \mu \mathrm{m} \\
\eta_{m} \rightarrow(1+j) \sqrt{\frac{\pi \mu_{m} f}{\sigma_{m}}}=(1+j) R_{s}=Z_{s} .
\end{gathered}
$$

The skin depth $\delta=0.66,0.21 \mu \mathrm{m}$ at $f=10,100 \mathrm{GHz}$ in copper. Thus, the assumption that $L, w \gg \delta$ holds if $w \geq 5$, $2 \mu \mathrm{m}$ at $f=10,100 \mathrm{GHz}$ because we are taking $L \geq w$.

\section{MODIFIED DyAdic GREEN's FunCTION FOR FINITE METAL THICKNESS}

Follow the procedure in (26) to get the $i$ th average, invoking (56)

$$
\begin{aligned}
& \left\langle\tilde{\mathbf{E}}_{c, \tan }(n, y)\right\rangle_{\mathrm{av}, i}=\left\langle\tilde{\mathbf{E}}_{u i, \tan }, \tilde{\mathbf{E}}_{l i, \tan }\right\rangle_{\mathrm{av}} \\
& =\left\langle\hat{y} \times\left[Z_{u u} \tilde{\mathbf{H}}_{s u i}+Z_{u l} \tilde{\mathbf{H}}_{s l i}\right],\right. \\
& \left.-\hat{y} \times\left[Z_{l u} \tilde{\mathbf{H}}_{s u i}+Z_{l l} \tilde{\mathbf{H}}_{s l i}\right]\right\rangle_{\text {av }} \\
& =\left\langle Z_{u u} \hat{y} \times\left[\begin{array}{c}
-\tilde{J}_{s u z i} \\
\tilde{J}_{s u x i}
\end{array}\right]+Z_{u l} \hat{y}\right. \\
& \times\left[\begin{array}{c}
\tilde{J}_{s l z i} \\
-\tilde{J}_{s l x i}
\end{array}\right],-Z_{l u} \hat{y} \times\left[\begin{array}{c}
-\tilde{J}_{s u z i} \\
\tilde{J}_{s u x i}
\end{array}\right] \\
& \left.-Z_{l l} \hat{y} \times\left[\begin{array}{c}
\tilde{J}_{s l z i} \\
-\widetilde{J}_{s l x i}
\end{array}\right]\right\rangle_{\text {av }} \\
& =\left\langle Z_{u u} \tilde{\mathbf{J}}_{s u i}-Z_{u l} \tilde{\mathbf{J}}_{s l i},-Z_{l u} \tilde{\mathbf{J}}_{s u i}\right. \\
& \left.+Z_{l l} \tilde{\mathbf{J}}_{s l i}\right\rangle_{\mathbf{a v}} \text {. }
\end{aligned}
$$

Then use (25) to obtain (59), shown at the bottom of the following page. From (59), we may again create an improper 
dyadic Green's function. Consider a limiting case when we take $\tilde{J}_{s u i} \rightarrow 0$, not an unreasonable assignment for a strip configuration when the preponderance on the material is in a substrate form. (In symmetric stripline this is an invalid assignment.) Equation (59) reduces to

$$
\begin{aligned}
\left\langle\tilde{\mathbf{E}}_{c, \tan }(n, y)\right\rangle_{\mathrm{av}, i} & =\frac{Z_{l l}\left|Z_{l l}\right|-Z_{u l}\left|Z_{u l}\right|}{\left|Z_{l l}\right|+\left|Z_{u l}\right|}\left[\begin{array}{c}
\tilde{J}_{s l x i} \\
\tilde{J}_{s l z i}
\end{array}\right] \\
& =\frac{Z_{l l}\left|Z_{l l}\right|-Z_{u l}\left|Z_{u l}\right|}{\left|Z_{l l}\right|+\left|Z_{u l}\right|} \tilde{\mathbf{J}}_{s l i} .
\end{aligned}
$$

Inserting (56c) and (57) into (60), noting that $R_{s}=1 /\left(\sigma_{m} \delta\right)$, the coefficient in (60) is shown in (61), at the bottom of this page.

If instead of using (25) we go back to (58), and allow the upper surface current to be zero

$$
\begin{aligned}
\left\langle\tilde{\mathbf{E}}_{c, \tan }(n, y)\right\rangle_{\mathrm{av}, i} \\
=\left\langle\tilde{\mathbf{E}}_{u i, \tan }, \tilde{\mathbf{E}}_{l i, \tan }\right\rangle_{\mathrm{av}} \\
=\left\langle\hat{y} \times Z_{u l} \tilde{\mathbf{H}}_{s l i},-\hat{y} \times Z_{l l} \tilde{\mathbf{H}}_{s l i}\right\rangle_{\mathrm{av}} \tilde{J}_{s l z i} \\
=\left\langle Z_{u l} \hat{y} \times\left[\begin{array}{c}
\tilde{J}_{s l z i} \\
-\widetilde{J}_{s l x i}
\end{array}\right],-Z_{l l} \hat{y} \times\left[\begin{array}{c}
\tilde{J}_{s l x i} \\
-\widetilde{J}_{\mathrm{av}}
\end{array}\right.\right. \\
=\left\langle-Z_{u l} \tilde{\mathbf{J}}_{s l i}, Z_{l l} \tilde{\mathbf{J}}_{s l i}\right\rangle_{\mathrm{av}}
\end{aligned}
$$

and evaluate the averaging operator as a simple sum (not a weight), we find

$$
\left\langle\tilde{\mathbf{E}}_{c, \tan }(n, y)\right\rangle_{\mathrm{av}, i}=\left(Z_{l l}-Z_{u l}\right) \tilde{\mathbf{J}}_{s i} .
$$

This gives

$$
C_{Z}=\frac{1}{\sigma_{m} t} \frac{\frac{(1+j) t}{\delta}}{\tanh \left[\frac{(1+j) t}{\delta}\right]}\left\{1+\frac{1}{\cosh \left[\frac{(1+j) t}{\delta}\right]}\right\} .
$$

Finally, if we neglect the nondiagonal matrix element $Z_{u l}$ in the field impedance (56c)

$$
C_{Z}=\frac{1}{\sigma_{m} t} \frac{\frac{(1+j) t}{\delta}}{\tanh \left[\frac{(1+j) t}{\delta}\right]} .
$$

Form (65) is like that seen in [24] and gives similar $f$ variation to $[25]^{3}$. The three cases in (61), (64), and (65) generate the three modified dyadic Green's functions (note that $\widetilde{\mathbf{J}}_{s l i} \rightarrow \tilde{\mathbf{J}}_{s i}$ ), shown in (66)-(68), at the bottom of this page.

${ }^{3}$ In [25], note that $t=0.5 \mu \mathrm{m}$ and $\rho_{c}=2.44 \times 10^{-6} \Omega \cdot \mathrm{cm}=2.44 \mu \Omega$.

$$
\begin{aligned}
\left\langle\tilde{\mathbf{E}}_{c, \tan }(n, y)\right\rangle_{\mathrm{av}, i}=\frac{\left(Z_{u u} \tilde{J}_{s u x i}-Z_{u l} \tilde{J}_{s l x i}\right)\left|Z_{u u} \tilde{J}_{s u x i}-Z_{u l} \tilde{J}_{s l x i}\right|+\left(Z_{l l} \tilde{J}_{s l x i}-Z_{l u} \tilde{J}_{s u x i}\right)\left|Z_{l l} \tilde{J}_{s l x i}-Z_{l u} \tilde{J}_{s u x i}\right|}{\left|Z_{u u} \tilde{J}_{s u x i}-Z_{u l} \tilde{J}_{s l x i}\right|+\left|Z_{l l} \tilde{J}_{s l x i}-Z_{l u} \tilde{J}_{s u x i}\right|} \hat{x} & \\
+\frac{\left(Z_{u u} \tilde{J}_{s u z i}-Z_{u l} \tilde{J}_{s l z i}\right)\left|Z_{u u} \tilde{J}_{s u z i}-Z_{u l} \tilde{J}_{s l z i}\right|+\left(Z_{l l} \tilde{J}_{s l z i}-Z_{l u} \tilde{J}_{s u z i}\right)\left|Z_{l l} \tilde{J}_{s l z i}-Z_{l u} \tilde{J}_{s u z i}\right|}{\left|Z_{u u} \tilde{J}_{s u z i}-Z_{u l} \tilde{J}_{s l z i}\right|+\left|Z_{l l} \tilde{J}_{s l z i}-Z_{l u} \tilde{J}_{s u z i}\right|} & \hat{z}
\end{aligned}
$$

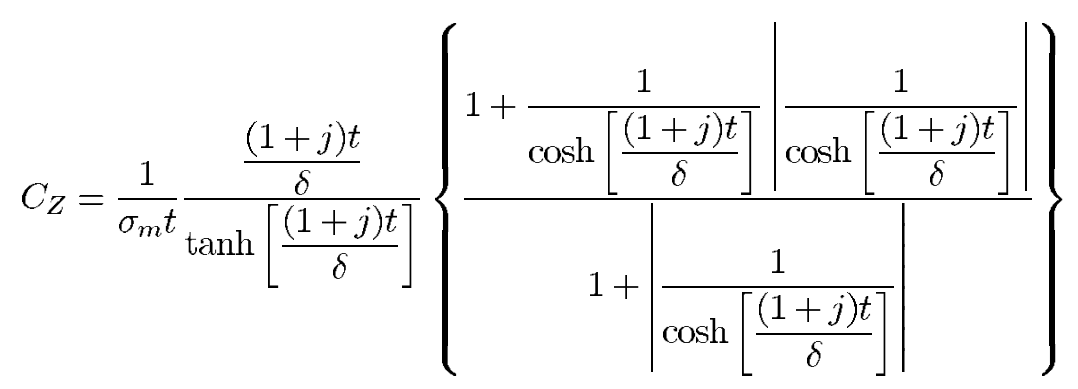

$$
\begin{aligned}
\mathbf{G}_{\mathbf{E}_{s} \mathbf{J}_{s i}}^{\prime} & =\left[\begin{array}{cc}
G_{\mathbf{E}_{s} \mathbf{J}_{s i} x x}-\frac{Z_{l l}\left|Z_{l l}\right|-Z_{u l}\left|Z_{u l}\right|}{\left|Z_{l l}\right|+\left|Z_{u l}\right|} & G_{\mathbf{E}_{s} \mathbf{J}_{s i} x z} \\
G_{\mathbf{E}_{s} \mathbf{J}_{s i} z x} & G_{\mathbf{E}_{s} \mathbf{J}_{s i} z z}-\frac{Z_{l l}\left|Z_{l l}\right|-Z_{u l}\left|Z_{u l}\right|}{\left|Z_{l l}\right|+\left|Z_{u l}\right|}
\end{array}\right] \\
\mathbf{G}_{\mathbf{E}_{s} \mathbf{J}_{s i}}^{\prime} & =\left[\begin{array}{cc}
G_{\mathbf{E}_{s} \mathbf{J}_{s i} x x}-\left(Z_{l l}-Z_{u l}\right) & G_{\mathbf{E}_{s} \mathbf{J}_{s i} x z} \\
G_{\mathbf{E}_{s} \mathbf{J}_{s i} z x} & G_{\mathbf{E}_{s} \mathbf{J}_{s i} z z}-\left(Z_{l l}-Z_{u l}\right)
\end{array}\right] \\
\mathbf{G}_{\mathbf{E}_{s} \mathbf{J}_{s i}}^{\prime} & =\left[\begin{array}{cc}
G_{\mathbf{E}_{s} \mathbf{s}_{s i} x x}-Z_{l l} & G_{\mathbf{E}_{s} \mathbf{J}_{s i} x z} \\
G_{\mathbf{E}_{s} \mathbf{J}_{s i} z x} & G_{\mathbf{E}_{s} \mathbf{J}_{s i} z z}-Z_{l l}
\end{array}\right]
\end{aligned}
$$




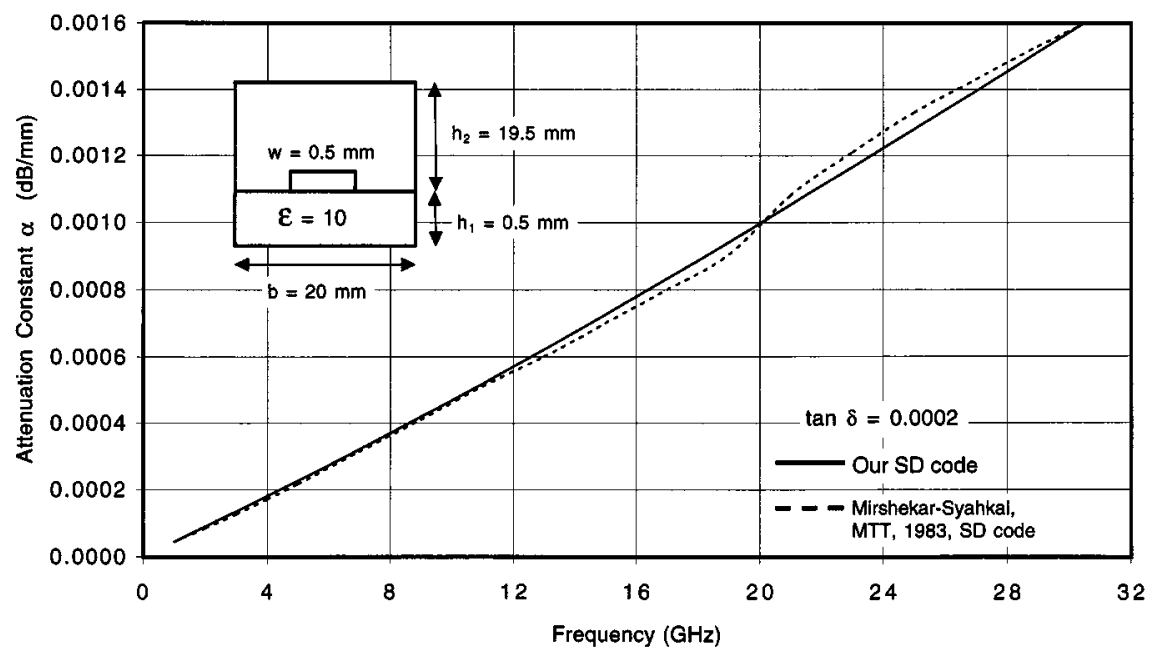

Fig. 2. Attenuation constant for dielectric loss versus frequency for $\tan \delta=2.0 \times 10^{-4}$. The code (basis function number $n_{x}=n_{z}=3$ and spectral number $n=200$ ) was compared to [27] (note that, in this reference, an approximate two-term expansion was used for surface current $J_{s z}$ with $J_{s x}=0$ ), which is also a full-wave calculation. Microstrip structure has width $=0.500 \mathrm{~mm}$, substrate height $=0.500 \mathrm{~mm}$, relative dielectric constant $=10.0$, box width $=20 \mathrm{~mm}$, and box height above substrate (air region) $=19.5 \mathrm{~mm}$.

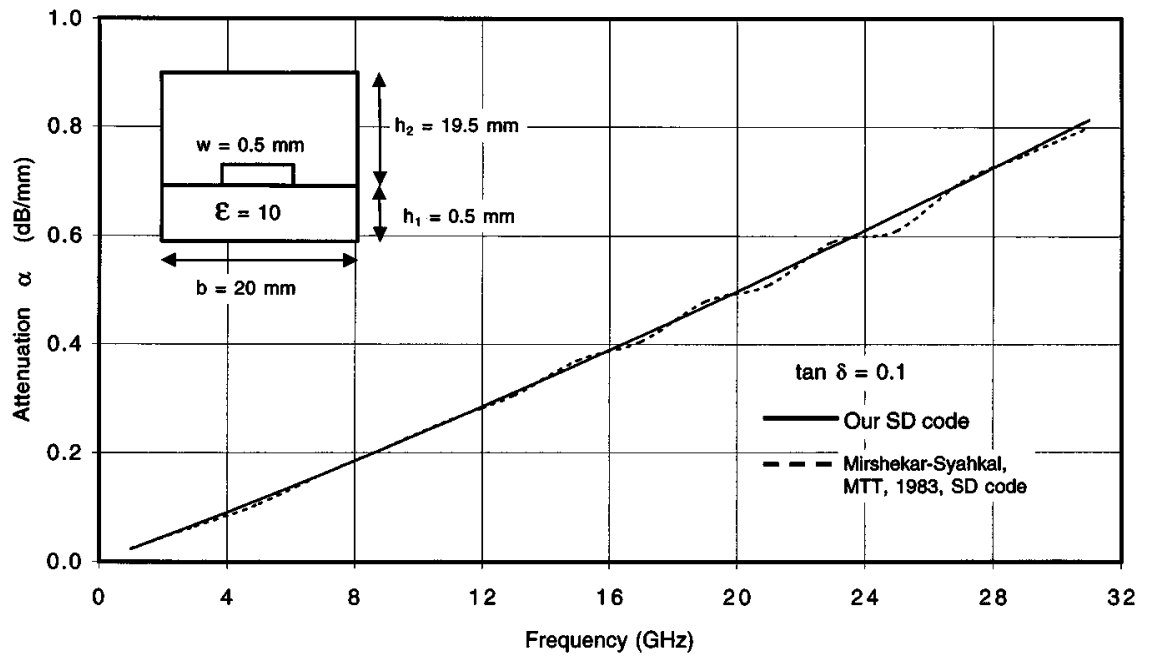

Fig. 3. Attenuation constant for dielectric loss versus frequency for $\tan \delta=1.0 \times 10^{-1}$. All other parameters are the same as in Fig. 2 .

There are three regimes of metal thickness: $t \gg \delta, t \approx \delta$, $t \ll \delta$. When $t \gg \delta$, the sinh and cosh functions approach $\infty$ and tanh approaches one. This makes

$$
\lim _{t / \delta \rightarrow \infty} C_{Z} \rightarrow \frac{1}{\sigma_{m} \delta}
$$

for all formulas (61), (64), and (65). In the other extreme when $t \ll \delta$

$$
\lim _{t / \delta \rightarrow 0} C_{Z} \rightarrow \frac{1}{\sigma_{m} t}\left\{\begin{array}{ll}
1 ; & (60) \\
2 ; & (63) \\
1 ; & (64)
\end{array}\right\} .
$$

It is clear from (70) that (61) and (65) may be the preferable ones to employ because of the unity limit expected for $t / \delta \rightarrow 0$.

\section{NUMERICAL RESUlTS FOR THE ANALYTICAL Limiting MODIFIED GREEN's FUNCTION FORMULAS}

Although we are particularly interested in applying the results here to highly anisotropic layered guiding structures, it is possible to validate some of the concepts here in a much simpler system. We choose microstrip over a GaAs substrate, which was measured for various geometric dimensions over the nominal 5-40-GHz frequency range [26]. To compare our theoretical dyadic Green's function modification work to experiment, we must also include dielectric loss to obtain the total loss.

In order to test our anisotropic Green's function SD code for dielectric-loss evaluation, the code (basis function number $n_{x}=n_{z}=3$ and spectral number $n=200$ ) was compared to [27], which is also a full-wave calculation. Test case was microstrip with width $2 w=0.500 \mathrm{~mm}$, substrate height $d=0.500 \mathrm{~mm}$, relative dielectric constant $\varepsilon_{r 1}=10.0$, box width $=2 a=20 \mathrm{~mm}$, and box height above substrate (air region) $=h=19.5 \mathrm{~mm}$ (notation used from [27]—note that, in this reference, an approximate two-term expansion was used for surface current $J_{s z}$ with $J_{s x}=0$ ). Two loss tangent cases were investigated, i.e., $\tan \delta=2.0 \times 10^{-4}$ and $1.0 \times 10^{-1}$, which are shown in Figs. 2 and $3\left(\varepsilon_{\text {diel }}=\varepsilon_{r}-j \varepsilon_{r} \tan \delta, \varepsilon_{r}=\right.$ real part, and $\tan \delta=\sigma_{d} / \omega \varepsilon_{r}$ for volumetric conductive dielectric loss). 


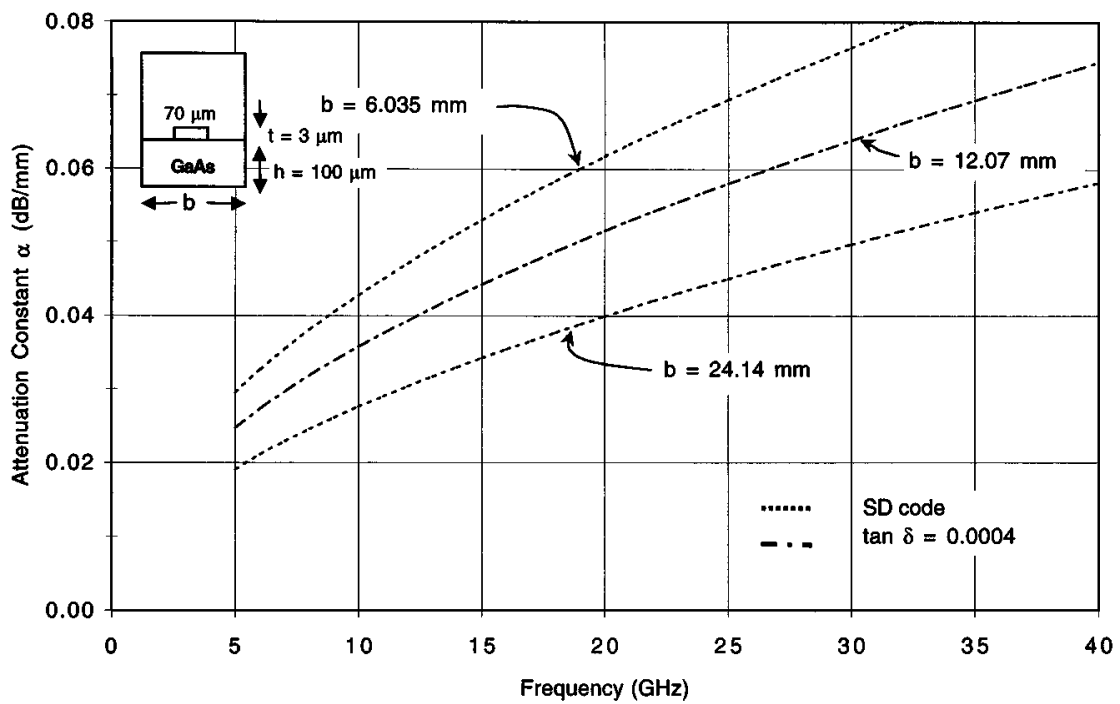

Fig. 4. Attenuation constant for microstrip versus frequency for varying box width $b$. Loss tangent held to tan $\delta=1.0 \times 10^{-4}$ and conductor loss included using (65). Structure is a 70- $\mu \mathrm{m}$-wide microstrip, with substrate height $100 \mu \mathrm{m}$, relative dielectric constant $\varepsilon_{r}=12.9$, box height above substrate (air region) $10 \mathrm{~mm}$, metal thickness $t=3 \mu \mathrm{m}$ and conductivity $\sigma_{s}=4.1 \times 10^{7} \mathrm{~S} / \mathrm{m}$ (gold). $n_{x}=n_{z}=4$ and $n=300$.

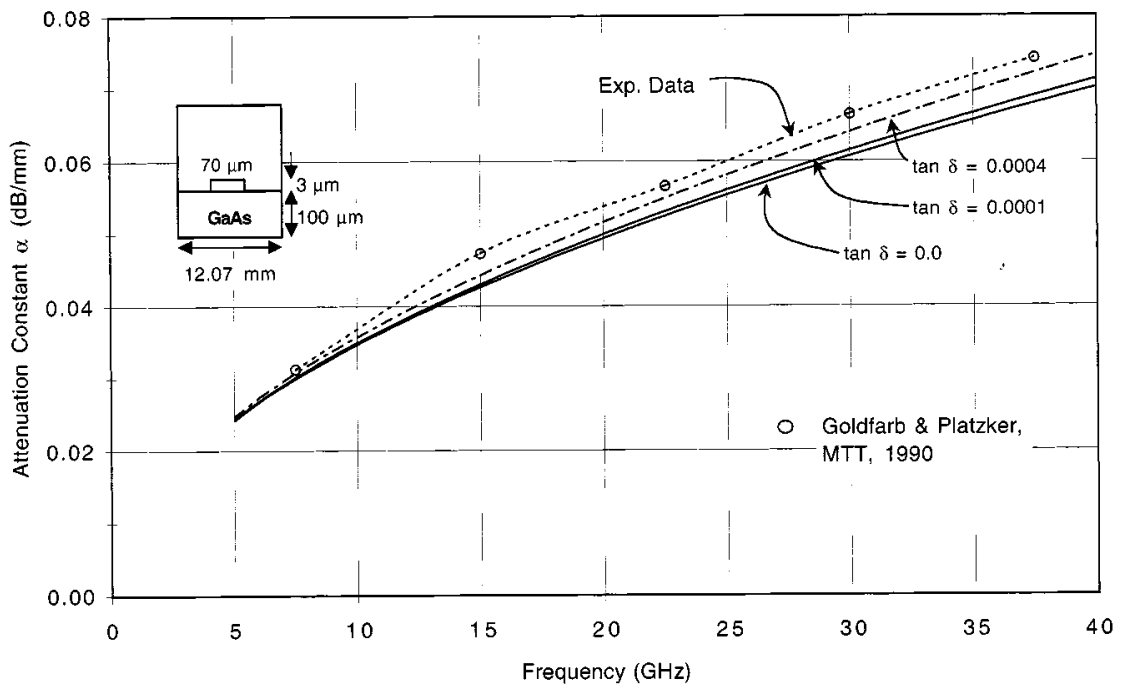

Fig. 5. Attenuation constant for microstrip versus frequency for varying loss tangent using (65) for conductor effect. Width $b=12.07 \mathrm{~mm}$, with all other parameters the same as in Fig. 4. Reference [26] experimental data are shown.

Slight waviness in the reference's curves are due to reading off the data, but agreement with our code results is excellent, being within $1 \%$, and allowing us to proceed on to the total loss calculation.

Now return to the experimental microstrip cases. First consider a $w=70 \mu \mathrm{m}$ wide microstrip, with substrate height $h_{s}=100 \mu \mathrm{m}$, relative dielectric constant $\varepsilon_{r}=12.9$, box height above substrate (air region) $=h_{a}=10 \mathrm{~mm}$, and varying box width $=b=6.035,12.07,24.14 \mathrm{~mm}$. The latter two dimensions, i.e., $h_{a}$ and $b$, are not constrained directly by the laboratory setup since an enclosure was not used. However, being completely open on top allows $h_{a}$ to be chosen large enough to avoid propagation constant sensitivity. Fig. 4 shows the dependence of attenuation $\alpha$ on $b$ using (65) for conductor strip loss. [This result is also the common factor in (61) and (64).] The middle result is the one we will use for $b$ since it best cor- responds to the substrate extent. This $b$ choice is also consistent with [28], which also looked at that case. Notice the sensitivity of $\alpha$ on $b$. On the other hand, $\beta(\gamma=\alpha+j \beta=$ total propagation constant), only weakly depends on $b$. Using $b=12.07 \mathrm{~mm}$, Fig. 5 plots the experimental and theoretical $w=70 \mathrm{~mm}$ results for varying loss tangent $\left(n_{x}=n_{z}=4\right.$ and $\left.n=300\right)$, with metal thickness $t=3 \mu \mathrm{m}$ and conductivity $\sigma_{s}=4.1 \times 10^{7} \mathrm{~S} / \mathrm{m}$ (mostly gold). Loss tangent $\tan \delta=4 \times 10^{-4}$ may best describe the substrate gallium-arsenide material. Since we have not taken into account either surface roughness or ground plane (12- $\mu \mathrm{m}$ thick $\mathrm{Au}$ ) conductor loss, both of which should be very tiny, this agreement, which is within a few percent, is considered to be good.

Fig. 6 compares theory and experiment for the $w=10 \mu \mathrm{m}$ case. At and below $f=25 \mathrm{GHz}$, agreement is excellent $\left(\tan \delta=4 \times 10^{-4}\right)$; above this frequency, the data is suspect. 


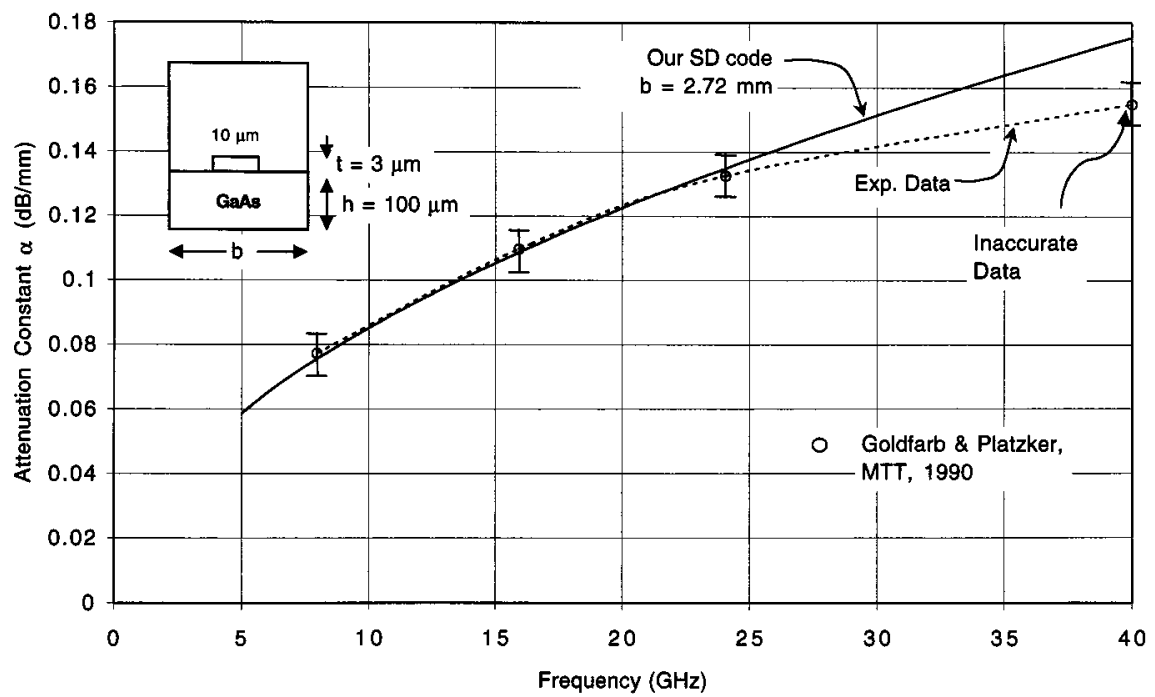

Fig. 6. Comparison of theoretical and experimental [26] attenuation constant for the $w=10 \mu \mathrm{m}$ microstrip case.

TABLE I

Attenuation CONSTANT $\alpha$ (In Decibels PER Millimeter) BASED UPON DYADIC GREEN'S FUNCTION MODIFICATION FORMULAS $C_{Z}$ (NO DIELECTRIC LOSS)

\begin{tabular}{lccc}
\hline & & $C_{Z}$ Formulas \\
$f(\mathrm{GHz})$ & $(65)$ & $(61)$ & $(64)$ \\
\hline 5 & 0.0242154 & $0.0212510(-12.24 \%)$ & $0.022789(-5.89 \%)$ \\
10 & 0.0346546 & $0.0331317(-4.40 \%)$ & $0.032518(-6.17 \%)$ \\
15 & 0.0425785 & $0.0417952(-1.84 \%)$ & $0.041741(-1.97 \%)$ \\
20 & 0.0493136 & $0.0488761(-0.887 \%)$ & $0.049240(0.149 \%)$ \\
25 & 0.0552480 & $0.0549882(-0.470 \%)$ & $0.055434(0.337 \%)$ \\
30 & 0.0606049 & $0.0604447(-0.264 \%)$ & $0.060810(0.338 \%)$ \\
35 & 0.0655182 & $0.0654161(-0.156 \%)$ & $0.065665(0.224 \%)$ \\
40 & 0.0700740 & $0.0700069(-0.0958 \%)$ & $0.070156(0.117 \%)$ \\
\hline
\end{tabular}

Due to this, we use the $w=70 \mu \mathrm{m}$ case to examine the other Green's function modification formulas [see (61) and (64)]. All three formulas are compared in Table I, under no dielectric loss, as to how they affect the attenuation constant. Comparison in this table is reasonable because all of the formulas produce very similar numerical values, although they have slightly different dependencies with frequency. Results given for $\alpha$ (in decibels per millimeter) in parentheses using $C_{Z}$ formulas (61) and (64) are percentage deviations from $\alpha$ using (65). It is seen that $\alpha$ using (61) is slightly lower than $\alpha$ using (65), the deviation getting less as the frequency increases. $\alpha$ using (64), in comparison to $\alpha$ using (65), is lower and gets nonmonotonically closer as frequency increases, then it is higher after crossing $\alpha$ [using (65)] and begins to come closer again.

\section{CONCLUSIONS}

This paper has shown how to systematically develop the formalism for finding dyadic Green's function modifications for zero-thickness conductor SD codes when studying loss of mi- crostrip configurations. The hierarchy of formulas has at its bottom several that are analytically easy to evaluate when modifying anisotropic structure dyadic Green's functions. Philosophy presented here has recently been followed for anisotropic ferroelectric coplanar structures, allowing excellent description of experimental attenuation results [29], [30].

\section{ACKNOWLEDGMENT}

The author wishes to thank M. Daniel, DCS Corporation, Alexandria, VA, for his help with the software.

\section{REFERENCES}

[1] C. M. Krowne, "Theoretical considerations for full-wave electromagnetic-media interactions in layered structures with ferroelectric or ferromagnetic materials," Proc. SPIE-Int. Soc. Opt. Eng., vol. 4097, pp. 70-84, July 2000

[2] — "Theoretical considerations for finding anisotropic permittivity in layered ferroelectric/ferromagnetic structures from full-wave electromagnetic simulations," Microwave Opt. Technol. Lett., vol. 28, pp. 63-69, Jan.-Feb. 2001

[3] C. M. Krowne, S. W. Kirchoefer, and J. M. Pond, "Anisotropic permittivity extraction from phase propagation measurements using an anisotropic full-wave Green's function solver for coplanar ferroelectric thin film devices," in IEEE MTT-S Int. Microwave Symp. Dig., Boston, MA, June 11-16, 2000, pp. 1193-1196.

[4] C. M. Krowne, "Relationships for Green's function spectral dyadics involving anisotropic imperfect conductors imbedded in layered anisotropic media," IEEE Trans. Antennas Propagat, vol. 37, pp. 1207-1211, Sept. 1989.

[5] C. L. Holloway and E. F. Kuester, "Power loss associated with conducting and superconducting rough surfaces," IEEE Trans. Microwave Theory Tech., vol. 48, pp. 1601-1610, Oct. 2000.

[6] K. M. Coperich, A. C. Cangellaris, and A. E. Ruehli, "Rigorous modeling of the frequency dependence of ohmic losses in high-speed electrical interconnections," in IEEE Int. Electromagn. Compat. Symp. Dig., Washington, DC, Aug. 21-25, 2000, pp. 301-306.

[7] J.-F. Kiang, "Integral equation solution to the skin effect problem in conductor strips of finite thickness," IEEE Trans. Microwave Theory Tech., vol. 39, pp. 452-460, Mar. 1991.

[8] A. C. Cangellaris, "The importance of skin-effect in microstrip lines at high frequencies," in IEEE MTT-S Int. Microwave Symp. Dig., New York, NY, May 25-27, 1988, pp. 197-198.

[9] A. C. Cangellaris, J. L. Prince, and O. A. Palusinski, "Modeling of highspeed interconnects: An integral equation approach to inductance computation," in Proc. Summer Simulation Conf., Seattle, WA, July 25-28, 1988, pp. 33-36. 
[10] T. E. van Deventer, L. P. B. Katehi, and A. C. Cangellaris, "Analysis of conductor losses in high-speed interconnects," IEEE Trans. Microwave Theory Tech., vol. 42, pp. 78-83, Jan. 1994.

[11] T. E. van Deventer, P. B. Katehi, and A. C. Cangellaris, "An integral equation method for the evaluation of conductor and dielectric losses in high-frequency interconnects," IEEE Trans. Microwave Theory Tech., vol. 37, pp. 1964-1972, Dec. 1989

[12] D. Mirshekar-Syahkal and J. B. Davies, "Accurate solution of microstrip and coplanar structures for dispersion and for dielectric and conductor losses," IEEE Trans. Microwave Theory Tech., vol. MTT-27, pp. 694-699, July 1979.

[13] R. H. Jansen, "High-speed computation of single and coupled microstrip parameters including dispersion, high-order modes, loss, and finite strip thickness," IEEE Trans. Microwave Theory Tech., vol. MTT-26, pp. 75-82, Feb. 1978.

[14] J.-C. Liou and K. M. Lau, "Analysis of slow-wave transmission lines on multi-layered semiconductor structures including conductor loss," IEEE Trans. Microwave Theory Tech., vol. 41, pp. 824-829, May 1993.

[15] F. Olyslager, D. De Zutter, and K. Blomme, "Rigorous analysis of the propagation characteristics of general lossless and lossy multiconductor transmission lines in multilayered media," IEEE Trans. Microwave Theory Tech., vol. 41, pp. 79-88, Jan. 1993.

[16] J. Aguilera, R. Marques, and M. Horno, "Quasi-TEM surface impedance approaches for the analysis of MIC and MMIC transmission lines, including both conductor and substrate losses," IEEE Trans. Microwave Theory Tech., vol. 43, pp. 1553-1558, July 1995.

[17] G. B. Stracca, "A simple evaluation of losses in thin microstrips," IEEE Trans. Microwave Theory Tech., vol. 45, pp. 281-283, Feb. 1997.

[18] T. Takagi, B. Houshmand, and T. Itoh, "Practical metal loss implementation for a microstrip line structure using SIBC in FDTD simulation," in IEEE MTT-S Int. Microwave Symp. Dig., Denver, CO, June 8-13, 1997, pp. $1531-1534$.

[19] T. A. Lauer and I. Wolff, "A conducting sheet model for efficient wide band FDTD analysis of planar waveguide and circuits," in IEEE MTT-S Int. Microwave Symp. Dig., Anaheim, CA, June 13-19, 1997, pp. $1589-1592$

[20] T. Roy, T. K. Sarkar, and M. Swaminathan, "Surface integral formulation for calculating conductor and dielectric losses of various transmission structures," IEEE Trans. Microwave Theory Tech., vol. 43, pp. 176-185, Jan. 1995

[21] H. J. Finlay, R. H. Jansen, J. A. Jenkins, and I. G. Eddison, "Accurate characterization and modeling of transmission lines for GaAs MMIC's," IEEE Trans. Microwave Theory Tech., vol. 36, pp. 961-967, June 1988.

[22] N. K. Das and D. M. Pozar, "Full-wave spectral domain computation of material, radiation, and guided wave losses in infinite multi-layered printed transmission lines," IEEE Trans. Microwave Theory Tech., vol. 39, pp. 54-63, Jan. 1991

[23] C. M. Krowne, "Electromagnetic conductor loss inclusion method in self-consistent 2D Green's function solver for inhomogeneous microstrip ferrite circulator," Int. J. Numer. Modeling, vol. 12, no. 5, pp. 399-415, Sept.-Oct. 1999.

[24] E. Paleczny, D. Kinowski, J. F. Legier, P. Pribetich, and P. Kennis, "Comparison of full wave approaches for determination of microstrip conductor losses for MMIC applications," Electron. Lett., vol. 26, no. 25, pp. 2076-2077, Dec. 1990

[25] C. M. Krowne, "Microstrip conductor losses calculated by full wave and perturbational approaches," Electron. Lett., vol. 24, no. 9, pp. 552-553, Apr. 1988.

[26] M. E. Goldfarb and A. Platzker, "Losses in GaAs microstrip" IEEE Trans. Microwave Theory Tech., vol. 35, pp. 1957-1963, Dec. 1990.
[27] D. Mirshekar-Syahkal, "An accurate determination of dielectric loss effect in monolithic microwave integrated circuits including microstrip and coupled microstrip lines," IEEE Trans. Microwave Theory Tech., vol. MTT-31, pp. 950-954, Nov. 1983.

[28] J.-T. Kuo and T. Itoh, "Hybrid-mode computation of propagation and attenuation characteristics of parallel coupled microstrips with finite metallization thickness," IEEE Trans. Microwave Theory Tech., vol. 45, pp. 274-280, Feb. 1997.

[29] C. M. Krowne, M. Daniel, S. W. Kirchoefer, and J. M. Pond, "Anisotropic permittivity and attenuation extraction from phase propagation measurements using an anisotropic full-wave Green's function solver for coplanar ferroelectric thin film devices," IEEE Trans. Microwave Theory Tech., to be published.

[30] C. M. Krowne, "Dyadic Green's function coplanar metal modification term for attenuation of ferroelectric devices," presented at the DARPA FAME Workshop, Fort Lauderdale, FL, Oct. 11-13, 2000.

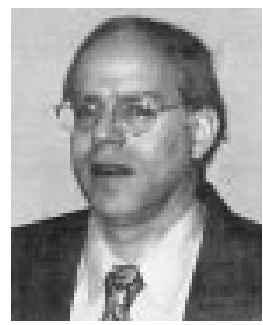

Clifford M. Krowne (S'73-M'74-SM'83) received the B.S. degree in physics from the University of California at Berkeley and Davis, in 1970, and the M.S. degree and Ph.D. degree (solid-state transport, many body scattering) from the University of California at Los Angeles, in 1972 and 1975, respectively.

He was with the Microelectronics Division, Lockheed Missiles and Space Company, Sunnyvale, CA. He was a Member of the Solid-State Technical Staff of the Watkins-Johnson Company, Palo Alto, CA. He was a faculty member in the Department of Electrical Engineering, North Carolina State University, Raleigh. He was also an Adjunct Professor of electrical engineering at the University of Maryland at College Park. Since 1982, he has been with the Microwave Technology Branch, Electronics Science and Technology Division, Naval Research Laboratory, Washington, DC, where he has been involved in the study of microwave and millimeter-wave properties of active and passive solid-state devices. He has authored or co-authored 170 conference and journal papers in solid-state electronics, microwave circuits, electromagnetics, and physics. He holds several patents. He has also written major portions of four books in the "Advances in Imaging and Electron Physics Series" on propagation in anisotropic media and circulation behavior in nonreciprocating media (New York: Academic, 1995, vol. 92; 1996, vol. 98; 1998, vol. 103; 1999, vol. 106), and has also made a contribution on numerical modeling of microstrip circulators in the ferrite devices area for the Electrical and Electronics Engineering Encyclopedia (New York: Wiley, 1999). He is listed in Who's Who in Frontiers of Science and Technology, Who's Who in the World, and Who's Who in Electromagnetics.

Dr. Krowne is a member of Phi Kappa Phi, Tau Beta Pi, and the American Physical Society. He is a Fellow of the Washington Academy of Sciences. He has served on the Technical Program Conference Committees of the IEEE Antennas and Propagation Society (IEEE AP-S) $(1983,1984)$ and the IEEE Microwave Theory and Techniques Society (IEEE MTT-S) (1982-1996). $\mathrm{He}$ has chaired sessions in the electromagnetic theory, microstrip antenna, and solid-state devices/circuits, superconductor, and monolithic circuit areas, and has organized two IEEE MTT-S workshops on two-dimensional (2-D)/three-dimensional (3-D) full-wave simulation (1992) and self-consistent particle transport/full-wave dynamic field simulation (1993). He was a member of the 1987 IEEE MTT-S Symposium Steering Committee. 